\title{
La Lingua Matematica: un'esperienza didattica nel Liceo Matematico
}

\section{The mathematical language: a teaching experience in Liceo Matematico}

\author{
Giuseppa Rita Cirmi, Salvatore D'Asero e Maria Flavia Mammana \\ Dipartimento di Matematica e Informatica, Università di Catania - Italia \\ 凶cirmi@dmi.unict.it, dasero@dmi.unict.it, mariaflavia.mammana@unict.it
}

Sunto / In questo lavoro si presenta un modulo didattico dal titolo La Lingua Matematica, rivolto a studenti del primo anno di scuola secondaria di secondo grado.

Il modulo è stato presentato in classi di Liceo $\mathrm{Ma}$ tematico, ma può essere proposto anche in altri contesti. Esso ha un duplice obiettivo: introdurre questioni relative a matematica e linguaggio da un lato e introdurre i concetti di teorema e dimostrazione dall'altro. Il percorso ha permesso di introdurre nozioni di aritmetica modulare e ha condotto gli studenti a confrontarsi, elaborare ipotesi, produrre argomentazioni e generare nuovi teoremi.

Parole chiave: laboratorio di matematica; Liceo Matematico; Teaching for Robust Understanding; matematica e linguaggio; argomentare in matematica.
Abstract / In this paper we present a teaching module entitled The Mathematical Language, aimed at students of the first year of upper secondary school.

The module has been presented in Liceo Matematico classes, but it can also be proposed in other environments. It has a twofold objective: to relate mathematics and language on the one hand and to introduce the concept of theorem and demonstration on the other. The module allowed to introduce some notions concerning modular arithmetic and led students to confront themselves, develop hypotheses, produce arguments and generate new theorems.

Keywords: mathematical laboratory; Liceo Matematico Project; Teaching for Robust Understanding; mathematics and language; mathematics argumentation.

\section{Introduzione}

II progetto Liceo Matematico nasce nel 2014 come progetto di ricerca del Dipartimento di Matematica dell'Università di Salerno. Pilastri del progetto sono interdisciplinarità, attività didattica laboratoriale, percorsi didattici in cui affrontare temi matematici che non hanno ancora trovato posto nel curriculum (Capone et al., 2017).

Sviluppatosi su tutto il territorio nazionale, ad oggi aderiscono al progetto 20 sedi universitarie (Poli) che collaborano, complessivamente, con circa 130 scuole secondarie superiori. Le scuole che aderiscono al progetto individuano al loro interno una o più sezioni (d'ora in avanti denominate Licei Ma-

(C) 2021 Cirmi Giuseppa Rita, D'Asero Salvatore e Mammana Maria Flavia. Questo è un articolo Open Access, sottoposto a un processo di revisione tra pari a doppio cieco, pubblicato dal Centro competenze didattica della matematica e dal Servizio comunicazione del DFA-SUPSI in collaborazione con il DECS. L'articolo è distribuito sotto i termini della Licenza Creative Commons Attribuzione 4.0 Internazionale (CC BY 4.0) che permette di usare, condividere e modificare l'articolo su qualsiasi mezzo a patto che I'autore e la fonte originale siano citati. 
tematici) in cui svolgere ore aggiuntive dedicate alla realizzazione di attività didattiche co-progettate da docenti della scuola e del Polo universitario di riferimento.

Le attività proposte in classe mirano a sviluppare capacità critiche e attitudine alla ricerca scientifica degli studenti, e a completare, in senso interdisciplinare, la loro formazione come cittadini e come persone.

II modulo che presentiamo in questo articolo è stato disegnato all'interno del Polo dell'Università di Catania per i Licei Matematici della Sicilia Orientale che afferiscono ad esso.

Il Polo di Catania nasce nel 2017 in seno al Dipartimento di Matematica e Informatica (DMI) e ha un'offerta formativa ormai giunta al suo quarto anno. Aderiscono al progetto tredici istituti di istruzione secondaria superiore, dodici licei e un istituto tecnico aeronautico.

I docenti dei Licei Matematici partecipano a incontri pomeridiani organizzati dal DMI, con cadenza quindicinale, in cui discutono e co-progettano con i docenti universitari le attività/moduli da proporre in classe ai loro studenti.

La Lingua Matematica, oggetto di questo articolo, è il modulo con cui accogliamo gli studenti del primo anno del Liceo Matematico. Esso prende spunto da un gioco di strategia, introduce i concetti di teorema e di dimostrazione e mira a sviluppare negli studenti competenze argomentative e capacità critiche, mettendo in relazione questioni relative a matematica e linguaggio.

Dopo una breve illustrazione del quadro di riferimento che sottende alla costruzione dell'attività, si descriverà il percorso didattico e si analizzeranno alcuni risultati della relativa sperimentazione in classe.

\section{Quadro teorico}

Nel 2013 Schoenfeld propone il quadro di riferimento Teaching for Robust Understanding (TRU) (Schoenfeld, 2013, 2014; Schoenfeld \& the Teaching for Robust Understanding Project, 2016).

II quadro individua cinque dimensioni di seguito descritte (Figura 1), che rendono un contesto di insegnamento/apprendimento realmente efficace e che favoriscono una comprensione profonda da parte degli studenti, realizzando così una didattica ambiziosa, robusta.

\begin{tabular}{|c|c|c|c|c|}
\hline The Content & $\begin{array}{l}\text { Cognitive } \\
\text { Demand }\end{array}$ & $\begin{array}{l}\text { Equitable Access } \\
\text { to Content }\end{array}$ & $\begin{array}{l}\text { Agency, } \\
\text { Ownership, and } \\
\text { Identity }\end{array}$ & $\begin{array}{l}\text { Formative } \\
\text { Assessment }\end{array}$ \\
\hline $\begin{array}{l}\text { The extent to which } \\
\text { classroom activity } \\
\text { structures provide } \\
\text { opportunities for } \\
\text { students to become } \\
\text { knowledgeable, } \\
\text { flexible, and } \\
\text { resourceful } \\
\text { disciplinary } \\
\text { thinkers. Discussions } \\
\text { are focused and } \\
\text { coherent, providing } \\
\text { opportunities to } \\
\text { learn disciplinary } \\
\text { ideas, techniques, } \\
\text { and perspectives, } \\
\text { make connections, } \\
\text { and develop } \\
\text { productive } \\
\text { disciplinary habits of } \\
\text { mind. }\end{array}$ & $\begin{array}{l}\text { The extent to which } \\
\text { students have } \\
\text { opportunities to } \\
\text { grapple with and } \\
\text { make sense of } \\
\text { important } \\
\text { disciplinary ideas } \\
\text { and their use. } \\
\text { Students learn best } \\
\text { when they are } \\
\text { challenged in ways } \\
\text { that provide room } \\
\text { and support for } \\
\text { growth, with task } \\
\text { difficulty ranging } \\
\text { from moderate to } \\
\text { demanding. The } \\
\text { level of challenge } \\
\text { should be conducive } \\
\text { to what has been } \\
\text { called "productive } \\
\text { struggle." }\end{array}$ & $\begin{array}{l}\text { The extent to which } \\
\text { classroom activity } \\
\text { structures invite and } \\
\text { support the active } \\
\text { engagement of all } \\
\text { of the students in } \\
\text { the classroom with } \\
\text { the core disciplinary } \\
\text { content being } \\
\text { addressed by the } \\
\text { class. Classrooms in } \\
\text { which a small } \\
\text { number of students } \\
\text { get most of the "air } \\
\text { time" are not } \\
\text { equitable, no } \\
\text { matter how rich the } \\
\text { content: all students } \\
\text { need to be involved } \\
\text { in meaningful ways. }\end{array}$ & $\begin{array}{l}\text { The extent to which } \\
\text { students are provided } \\
\text { opportunities to "walk } \\
\text { the walk and talk the } \\
\text { talk" - to contribute to } \\
\text { conversations about } \\
\text { disciplinary ideas, to } \\
\text { build on others' ideas } \\
\text { and have others build } \\
\text { on theirs - in ways } \\
\text { that contribute to } \\
\text { their development of } \\
\text { agency (the } \\
\text { willingness to } \\
\text { engage), their } \\
\text { ownership over the } \\
\text { content, and the } \\
\text { development of } \\
\text { positive identities as } \\
\text { thinkers and learners. }\end{array}$ & $\begin{array}{l}\text { The extent to which } \\
\text { classroom activities } \\
\text { elicit student } \\
\text { thinking and } \\
\text { subsequent } \\
\text { interactions respond } \\
\text { to those ideas, } \\
\text { building on } \\
\text { productive } \\
\text { beginnings and } \\
\text { addressing emerging } \\
\text { misunderstandings. } \\
\text { Powerful instruction } \\
\text { "meets students } \\
\text { where they are" and } \\
\text { gives them } \\
\text { opportunities to } \\
\text { deepen their } \\
\text { understandings. }\end{array}$ \\
\hline
\end{tabular}

Figura 1. Dimensioni del quadro TRU (Schoenfeld, 2016). 
Il contenuto: Il contenuto deve essere ricco, deve cioè rendere gli studenti dei "pensatori", in grado di imparare le idee e le tecniche proprie della disciplina e anche di produrre connessioni e sviluppare abitudini mentali produttive. Gli studenti devono «afferrare il contenuto per affrontare ciò che chiamiamo una lotta produttiva» (Schoenfeld, video di apertura del sito dedicato al quadro di riferimento TRU, https://truframework.org/, traduzione degli autori).

Richiesta Cognitiva: Durante le attività gli studenti devono avere l'opportunità di affrontare e capire a fondo le idee importanti della disciplina. Essi imparano meglio quando affrontano sfide, che favoriscono le lotte produttive e che permettono loro di crescere, mediante attività che diventano via via sempre più impegnative.

Accesso equo ai contenuti: Le attività in classe devono essere progettate in modo da favorire il coinvolgimento di tutti gli studenti in attività sfidanti, senza limitare i contenuti matematici.

Tutti gli studenti devono essere coinvolti in modo significativo: le attività in cui solo un piccolo numero di studenti ottiene la maggior parte dei risultati non sono eque/inclusive.

Azione, padronanza, identità: È opportuno proporre agli studenti attività in cui possano mettersi in gioco, proponendo il loro punto di vista, contribuendo alle discussioni e costruendo il proprio sapere. "I can do this!", dice Schoenfeld nel video: gli studenti rafforzano la loro autostima e la loro identità disciplinare ("Io penso come uno scienziato!", "Io penso come un fisico!", "lo penso come un matematico!").

Valutazione Formativa: L'attività deve essere tarata sulle conoscenze dello studente: un'attività robusta "incontra gli studenti dove sono" e offre loro l'opportunità di approfondire le loro conoscenze.

In breve,

«se il contenuto è ricco, gli studenti si impegnano, ottengono idee brillanti, costruiscono sulle idee dei compagni, possono costruire identità positive con l'insegnante che adatta opportunamente il livello di istruzione per gli studenti che si impegnano in modo produttivo».

(Schoenfeld, video in https://truframework.org/, traduzione degli autori)

Le dimensioni del quadro TRU trovano ampio spazio nel laboratorio di matematica.

Il laboratorio di matematica è

"quello spazio fenomenologico dell'insegnamento apprendimento della matematica che si struttura attraverso l'uso di specifici strumenti tecnologici e di articolati processi di negoziazione e in cui la conoscenza matematica viene assoggettata a un nuovo ordine rappresentativo, operativo e sociale per essere riconfigurata in oggetto di investigazione e poter essere quindi più efficacemente insegnata e appresa».

(Chiappini, 2007, p. 10)

Il laboratorio di matematica è oggi molto diffuso e appare già nei curricula dell'Unione Matematica Italiana del 2004 (Anichini et al., 2004):

«ll laboratorio di matematica non è un luogo fisico diverso dalla classe, è piuttosto un insieme strutturato di attività volte alla costruzione di significati degli oggetti matematici. II laboratorio, quindi, coinvolge persone (studenti e insegnanti), strutture (aule, strumenti, organizzazione degli spazi e dei tempi), idee (progetti, piani di attività didattiche, sperimentazioni).

L'ambiente del laboratorio di matematica è in qualche modo assimilabile a quello della bottega rinascimentale, nella quale gli apprendisti imparavano facendo e vedendo fare, comunicando fra loro e con gli esperti.

La costruzione di significati, nel laboratorio di matematica, è strettamente legata, da una parte, 
all'uso degli strumenti utilizzati nelle varie attività, dall'altra, alle interazioni tra le persone che si sviluppano durante l'esercizio di tali attività».

(Anichini et al., 2004, p. 28)

In laboratorio quindi

«l'alunno è attivo, formula le proprie ipotesi e ne controlla le conseguenze, progetta e sperimenta, discute e argomenta le proprie scelte, impara a raccogliere dati, negozia e costruisce significati, porta a conclusioni temporanee e a nuove aperture la costruzione delle conoscenze personali e collettive».

(Ministero dell'istruzione, dell'università e della ricerca [MIUR], 2012, p. 50)

Il laboratorio di matematica quindi si presta bene a favorire una didattica ambiziosa e robusta: in esso gli studenti lavorano su un problema (Contenuto), che non deve essere né troppo facile perché rischierebbero di annoiarsi, né troppo difficile perché potrebbero scoraggiarsi (Richiesta cognitiva), confrontandosi con gli studenti di tutta la classe (Accesso equo ai contenuti), affermando e discutendo le proprie scelte (Azione, padronanza identità).

\section{Il percorso didattico: La Lingua Matematica}

La Lingua Matematica è un percorso didattico di tipo laboratoriale che mira ad avviare processi di argomentazione e dimostrazione, a introdurre il concetto di teorema in matematica. Inoltre, si ha I'occasione per riflettere su questioni relative a matematica e linguaggio, mettendo in evidenza che il linguaggio è lo strumento col quale comunichiamo qualcosa, un certo contenuto (Bramanti \& Travaglini, 2009). L'attività di argomentazione si snoda attraverso produzioni scritte che devono essere chiare, precise: sottolineiamo infatti che, se in una comunicazione orale possiamo porre domande a più riprese per essere certi di aver compreso, nella comunicazione a distanza, scritta per esempio, è necessario comprendere in maniera univoca un messaggio, un'istruzione, una spiegazione, cioè è necessario scrivere in modo semplice e non ambiguo.

Il percorso, rivolto a studenti delle prime classi di scuola secondaria di secondo grado, ${ }^{1}$ si articola in 12 attività divise in tre fasi. ${ }^{2}$

Fase 1: La corsa a 20: gioco e ricerca di strategie.

Fase 2: Formalizzazione delle strategie mediante teoremi e produzione delle relative dimostrazioni.

Fase 3: Produzione e comprensione di varianti de La corsa a 20.

\section{Fase 1:}

Il percorso prende spunto da La corsa a 20, un gioco di strategia proposto per la prima volta da Brousseau (1997). Come in tutti i giochi di strategia, anche qui la capacità che ha un giocatore di prendere decisioni ha un grande impatto nel determinare il risultato e la fortuna non ha un peso rilevante. Agisce un giocatore alla volta, mentre gli altri restano in attesa.

La corsa a 20 viene presentato agli studenti come un vero e proprio gioco da tavolo, con istruzioni, pedina e tabellone (Figura 2).

1. La scuola secondaria di secondo grado in Italia dura cinque anni e corrisponde all'ultimo anno di scuola media e alla scuola media superiore o scuole professionali nel Canton Ticino.

2. Al fine di non appesantire la descrizione si è scelto di riportare negli allegati alcuni approfondimenti relativi a singole attività. Per ulteriori informazioni è possibile contattare gli autori dell'articolo. 


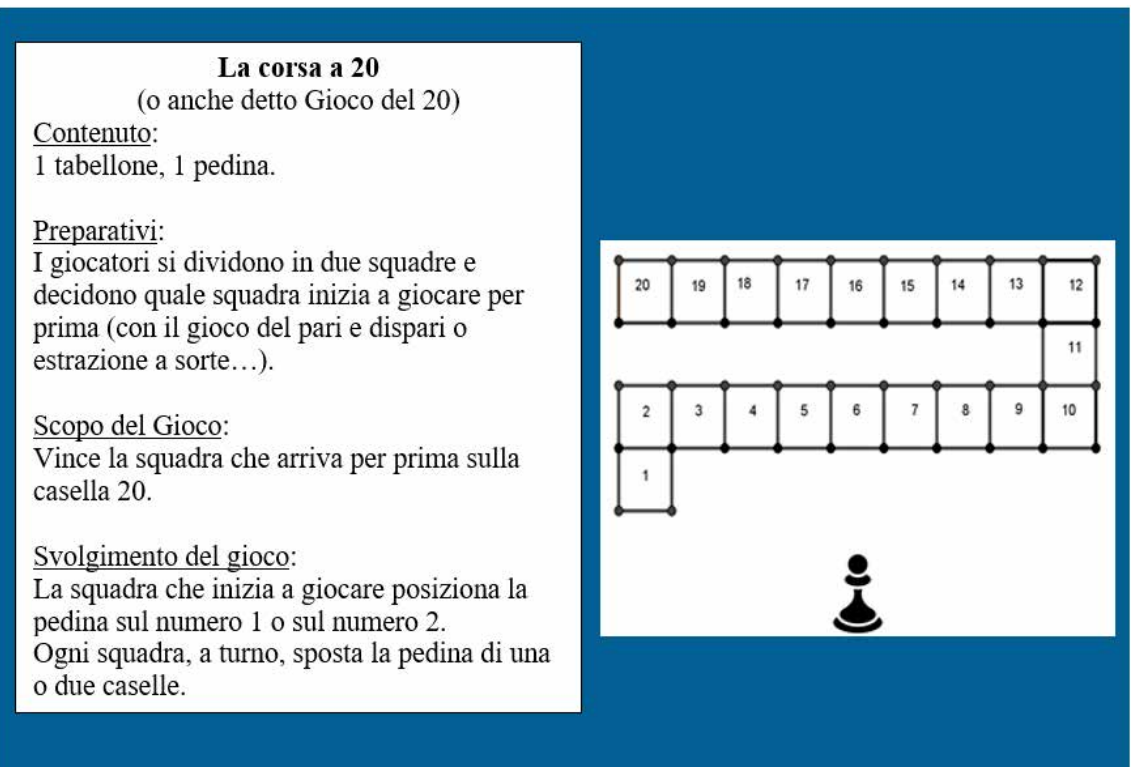

Figura 2. La corsa a 20: regole del gioco, tabellone e pedina.

Gli studenti, divisi in piccoli gruppi, sono invitati a giocare e a descrivere, per iscritto, la strategia vincente con le relative argomentazioni, (nell'Allegato 1 il lettore trova suggerimenti in merito alla strategia del gioco). A seguire i ragazzi, sempre in gruppi, giocano al Dico 29! (Figura 3). II gioco è simile a La corsa a 20. Questa volta però gli studenti sono invitati a giocare senza tabellone e pedina, ma alternandosi nel nominare il numero che vogliono raggiungere. Gli studenti elaborano la strategia vincente e argomentano la loro posizione (Allegato 1).

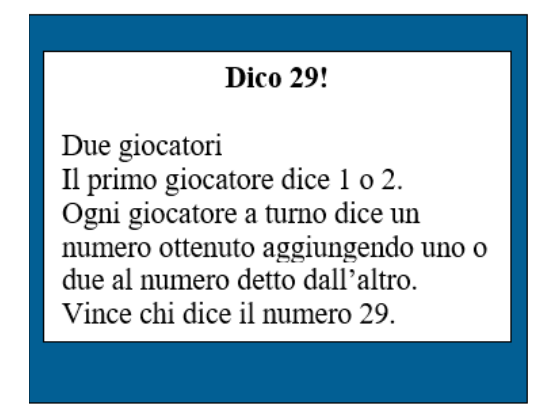

Figura 3. Dico 29!

Una discussione matematica (Bartolini Bussi et al., 1995), guidata dal docente, in cui si presentano e si confrontano le strategie trovate, chiude la Fase 1.

La revisione delle strategie nella discussione moderata dal docente si rivela sempre un momento intenso e di grande confronto.

Spesso infatti gli studenti, nelle descrizioni/argomentazioni delle loro strategie, danno per scontato alcune condizioni, o sono imprecisi:

Gruppo 1: «A inizio partita se gli avversari muovono di 1 la pedina dopo si deve muovere di 2; al contrario se gli avversari muovono di 2 bisogna muovere di 1 (prima delle caselle 12/13). Per vincere i giocatori, da queste due caselle, devono arrivare alla casella 17». 
Gruppo 2: «Bisogna conquistare le caselle n 5, 8, 11, 14, 17 per poter vincere.

PS: bisogna partire dal due.

Per vincere devi riuscire a far arrivare l'avversario alla casella $3 »$.

Gruppo 3: "Arrivare alla casella 14, perché se I'avversario si muove di una o due caselle (15 o 16) si va alla casella 17 anche se l'avversario muove di una o due (18 o 19) poi arrivando alla ventesima casella si vince».

Le proposte di questi tre gruppi mostrano ragionamenti piuttosto imprecisi e facilmente attaccabili o incompleti: non è detto che chi arriva alla casella 12 o 13 arrivi sicuramente alla 17 (Gruppo 1); oppure si può vincere senza partire dal 2 o senza che l'avversario passi per la casella 3 (Gruppo 2); infine, il ragionamento del Gruppo 3 è incompleto perché non individua caselle vincenti prima della quattordicesima.

Molto puntuale è stato il Gruppo 4 che ha riportato le mosse, ma che non è stato capace di operare una sintesi:

Gruppo 4: «Per vincere questo gioco bisogna iniziare sul numero 2. Poi l'avversario può muoversi nel 3 o nel 4, in ogni caso ti devi spostare sul 5; poi l'avversario si deve spostare sul 6 o sul 7. In ogni caso ti sposterai sull'8. Dopo l'avversario dovrà spostarsi sul 9 o 10. In ogni caso ti sposterai sul 14. poi l'avversario si sposterà sul 15 o 16 e tu in ogni caso ti sposterai sul 17. Poi l'avversario è costretto a mettersi sul 18 o 19 e tu hai vinto».

\section{Fase 2:}

Con la Fase 2 si inizia a passare dal linguaggio naturale al linguaggio matematico.

Si propongono le seguenti proposizioni (Tabella 1 ) chiedendo di indicarne il valore di verità, specificando che, per quelle proposizioni che si ritengono false, si deve portare un controesempio.

\begin{tabular}{|c|c|c|c|}
\hline & & V & $\mathrm{F}$ \\
\hline 1 & Se parto da 2 vinco. & & \\
\hline 2 & Se inizio per primo vinco. & & \\
\hline 3 & Se non parto da 2 non vinco. & & \\
\hline 4 & Se arrivo sulle caselle $2,5,8,11,14$, vinco. & & \\
\hline 5 & $\begin{array}{l}\text { Se avanzo di un numero di caselle diverso da quelle di cui avanza il } \\
\text { mio avversario vinco. }\end{array}$ & & \\
\hline 6 & $\begin{array}{l}\text { Se arrivo su una delle caselle } 2,5,8,11,14,17 \text { e avanzo di un } \\
\text { numero di caselle diverso da quelle di cui avanza il mio avversario } \\
\text { vinco. }\end{array}$ & & \\
\hline 7 & Se arrivo sulla casella 19 perdo. & & \\
\hline
\end{tabular}

Tabella 1. Vero o Falso?

Si passa poi a osservare che ciascuna delle precedenti frasi è costituita da due proposizioni legate tra loro da un "Se" (esplicitato) e da un "allora" (implicito), si introducono le notazioni A $\Rightarrow$ B oppure $A \Rightarrow B$ e infine i delicati concetti di teorema e di dimostrazione: a tal proposito si analizzano teoremi del linguaggio naturale (Se Marco vive a Roma allora Marco vive in Italia) e teoremi propri della matematica (Se $n$ è un numero naturale multiplo di quattro allora $n$ è un numero naturale pari).

Alla luce di quanto imparato si analizzano poi le 7 proposizioni della Tabella 1, individuando nella 
proposizione 6 un teorema che gli studenti, mediante una attività guidata, sono invitati a dimostrare. La proposizione 6 diventa allora il Teorema del come faccio a vincere!

NOTA: Sono state elaborate due dimostrazioni (due attività guidate) del Teorema del come faccio a vincere: una utilizza la proprietà caratteristica delle caselle vincenti (essere un numero del tipo 3n-1), I'altra permette di introdurre le classi di resto modulo 3. II docente è libero di proporre alla classe l'una, I'altra o entrambe le dimostrazioni. Nell'Allegato 2 è possibile visionare una dimostrazione guidata.

\section{Fase 3:}

Con la Fase 3 si chiude il percorso proponendo nuovamente un momento ludico: si gioca con alcune varianti de La corsa a 20 e si chiede agli studenti di inventarne altre.

I nuovi giochi diventano poi occasione per creare nuovi teoremi.

\section{Considerazioni e varianti}

La parte più intrigante per gli studenti è indubbiamente quella in cui giocano, o meglio quella in cui inventano giochi nuovi, che portano a enunciare e dimostrare nuovi teoremi (Fase 3).

Riportiamo a seguire due varianti proposte dagli studenti e una variante proposta da un docente, che ci sono sembrate molto interessanti.

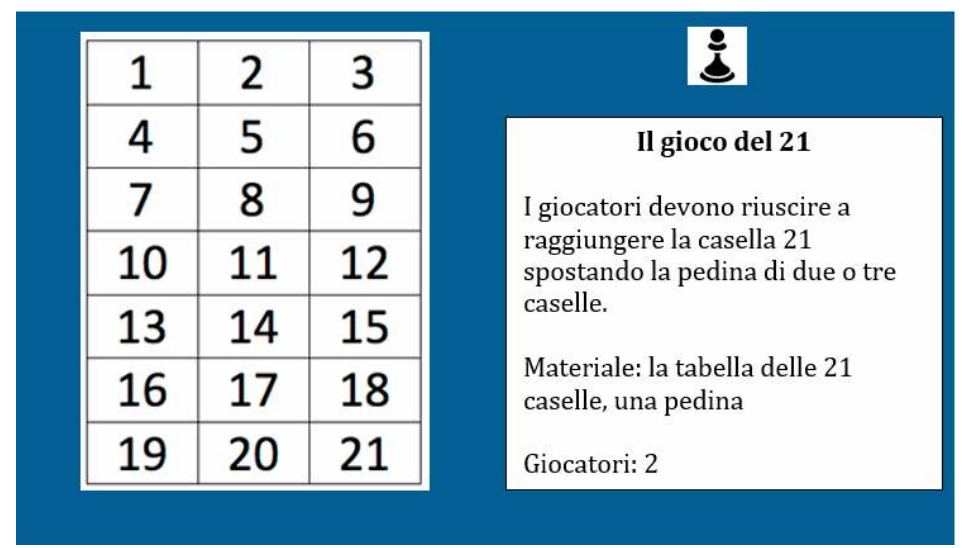

Figura 4. Il gioco del 21: tabellone, regole, pedina.

Un giocatore, anche non troppo esperto, si renderà conto che non sempre c'è un giocatore vincente, e la partita può anche finire in parità.

Caselle vincenti per questo gioco, cioè caselle che portano alla vittoria se ci si arriva e se si utilizza una corretta strategia sulle mosse da fare, sono 6, 11, 16.

Come suggerisce bene Gia., nella sua argomentazione:

Gia.: «Numeri vincenti sono 6-11-16-21.

In questo gioco possiamo adottare 2 strategie:

la prima strategia è quella vincente: conquistare per primi le caselle $6,11,16$ e dalla casella 16 in base a come muove l'avversario, si arriva alla casella 21 e si vince.

La seconda strategia è quella per pareggiare, quindi non c'è nessun vincitore. Evitando di fare arrivare l'avversario alle caselle vincenti». 
Gia. indica le caselle vincenti (che chiama numeri vincenti) e indica molto bene le "due strategie". La possibilità di pareggiare era proprio la novità che voleva introdurre $\mathrm{M}$.:

M.: «L'idea di questo gioco mi è venuta perché ho pensato se si potesse non solo vincere o perdere ma anche pareggiare».

A completamento del ragionamento indicato, A., L. e Ra. sottolineano che

A., L., Ra.: «Nell'elenco delle caselle vincenti non è incluso l'1 perché non è raggiungibile in alcun modo».

I ragazzi (anche M., autore del gioco), non avendo indicato le caselle utili per il posizionamento iniziale, hanno assunto che la pedina partisse dalla casella zero, non indicata sul tabellone.

La variante di Enn., Enr., Gio. e Ro., 14 anni (Figura 5).

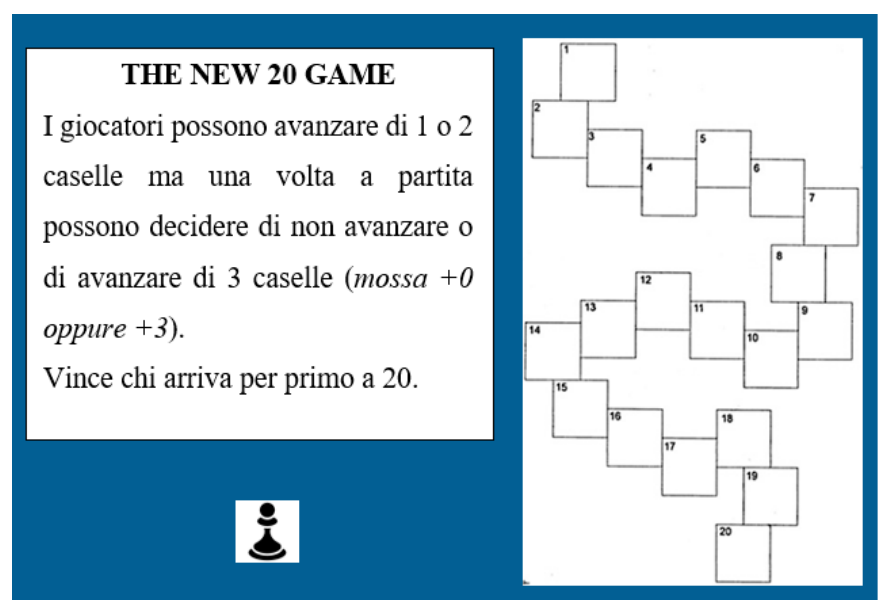

Figura 5. The new 20 game: Tabellone, regole, pedina.

Il The new 20 game è un gioco che, a fronte di un tabellone uguale, a meno della forma, a quello de La corsa a 20, presenta una novità nelle mosse: la possibilità, una volta a partita, di non avanzare o di avanzare di tre caselle. Questa novità pone delle possibilità di azione del giocatore più ampie e una strategia vincente un po' più complessa da elaborare.

Ragioniamo come segue.

Supponiamo di essere arrivati sulla casella 16 e di non avere mai utilizzato la mossa +0 oppure +3 che fa spostare la pedina di 0 o di 3 caselle. A prescindere dalla mossa dell'avversario io posso portarmi sulla casella 20 (Tabella 2).

\begin{tabular}{|l|l|l|}
\hline & Mossa dell'avversario & Mossa mia \\
\hline Possibilità 1 & +1 (casella 17) & +3 (casella 20) \\
\hline Possibilità 2 & +2 (casella 18) & +2 (casella 20) \\
\hline Possibilità 3 & +3 (casella 19) & +1 (casella 20) \\
\hline Possibilità 4 & +0 (casella 16) & +1 (casella 17) \\
\hline
\end{tabular}

Tabella 2. Possibili mosse a partire dalla casella 16. 
La possibilità 4 ci riporta a La corsa a 20. Infatti, a quel punto, I'avversario ha già utilizzato la mossa +0 oppure +3 e per lui il gioco si riduce a La corsa a 20. Quindi io avanzerò la pedina di due caselle se l'avversario la sposta di una e di una casella se l'avversario avanza di due, portandomi sulla casella 20. Andando a ritroso, la precedente casella vincente, se non ho ancora utilizzato la mossa +0 oppure +3 è la casella 13 (Tabella 3).

\begin{tabular}{|l|l|l|}
\hline & Mossa dell'avversario & Mossa mia \\
\hline Possibilità 1 & +1 (casella 14) & +2 (casella 16) \\
\hline Possibilità 2 & +2 (casella 15) & +1 (casella 16) \\
\hline Possibilità 3 & +3 (casella 16) & +1 (casella 17) \\
\hline Possibilità 4 & +0 (casella 13) & +1 (casella 14) \\
\hline
\end{tabular}

Tabella 3. Possibili mosse a partire dalla casella 13.

Se ci spostiamo secondo le possibilità 1 e 2 arriviamo alla casella 16 e ci troviamo nella condizione precedente. Se ci spostiamo secondo le possibilità 3 e 4 applichiamo la strategia de La corsa a 20. In ogni caso ci si riconduce ad un caso già studiato.

In generale, finché non viene usata la mossa speciale, le caselle vincenti saranno 1, 4, 7, 10, 13,16 (Figura 6). Quindi, se inizio per primo e mi sposto sulla casella 1 e procedo per mosse vincenti vinco la partita!

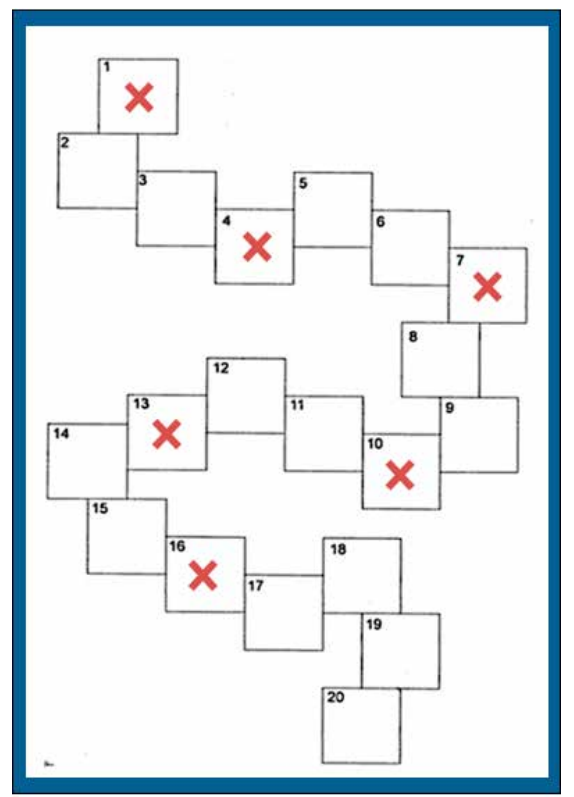

Figura 6. Caselle vincenti del The new 20 game.

II The new 20 game risulta essere un gioco piuttosto avvincente in cui entrano in gioco conoscenze pregresse. Probabilmente i quattro autori non avevano neanche inteso quanto avrebbero messo in difficoltà i compagni che si sono ritrovati a giocare con la loro variante.

La variante del Professore

La proposta del Professore prevede un tabellone costituito da caselle esagonali: due giocatori a tur- 
no spostano la stessa pedina dalla casella in cui si trova il cartoncino blu a quella in cui si trova il cartoncino rosso, procedendo da sinistra verso destra avanzando da una casella a una confinante in orizzontale o in diagonale - direzioni ovest-est, nord-est o sud-est (Figura 7).

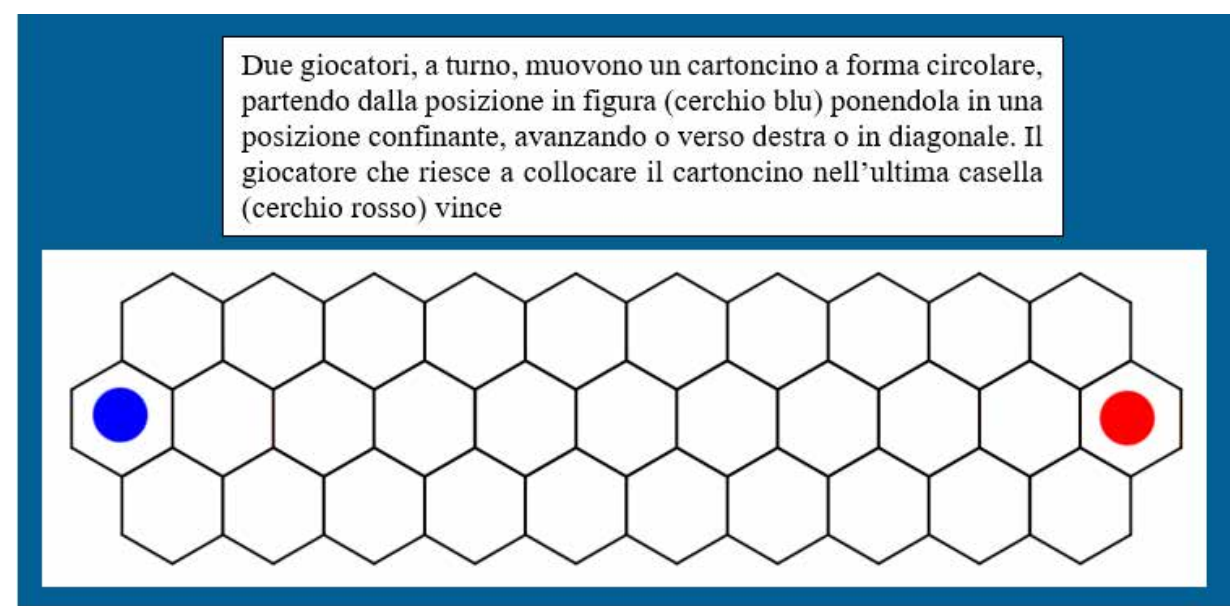

Figura 7. Il tabellone del gioco del Professore e le regole del gioco.

Se numeriamo in modo adeguato le caselle, l'equivalenza al gioco de La corsa a 20 apparirà chiaramente (Figura 8).

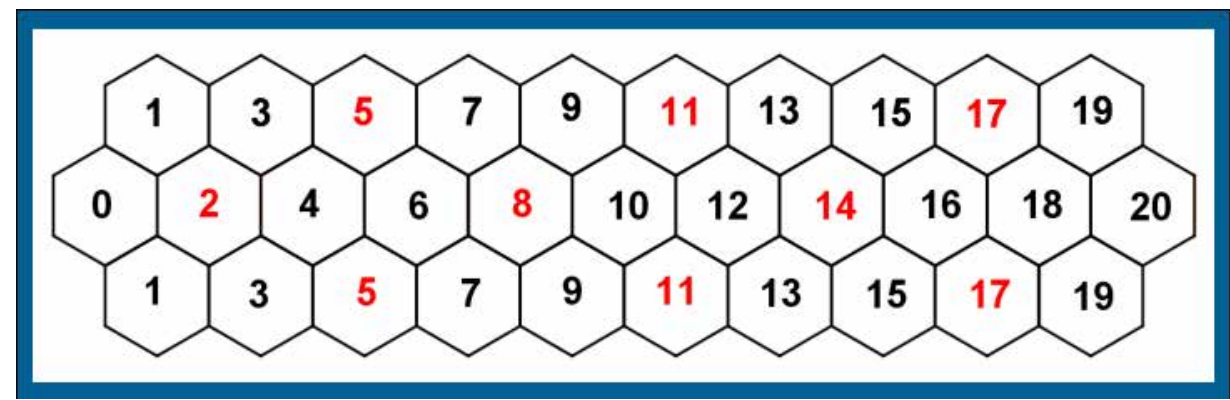

Figura 8. Numerazione del tabellone del gioco del Professore.

Avanzare di una casella in orizzontale equivale a fare un salto di due caselle ne La corsa a 20, avanzare di una casella in diagonale equivale a fare un salto di una casella ne La corsa a 20. Quindi, le caselle vincenti sono la 2, la 5, la 8, la 11, la 14, la 17 e la mossa vincente, trovandosi in una casella vincente, consiste nello spostarsi in una direzione diversa da quella di cui si è spostato l'avversario.

\section{Conclusioni e suggerimenti}

II percorso qui presentato prende spunto da un gioco da tavolo. II gioco accompagna diverse fasi dell'attività laboratoriale e si configura come strumento per attirare lo studente e avviare processi di conoscenza che si snoderanno attraverso discussioni, confronti, ragionamenti, dimostrazioni e nuovi giochi. D'altra parte, I'utilizzo di giochi nella didattica è sempre più diffuso. Si tratta di giochi che 
non hanno esclusivamente o principalmente uno scopo di intrattenimento, ma contengono elementi educativi: si pensi per esempio ai giochi seri, cioè videogiochi nati a scopi didattici in cui si impara in contesto (Sawyer, 2007), ai giochi di strategia noti a tutti come il gioco della Dama o degli Scacchi, che pur non nascendo come giochi educativi, sviluppano competenze, o alle favole che accompagnano attività didattiche dei più piccoli (Ponte, 2001).

L'utilizzo di giochi nella didattica permette di migliorare capacità di pensiero critico mentre si insegna un particolare argomento, permettendo agli studenti di pensare fuori dagli schemi mentre si seguono le regole (Zirawaga et al., 2017). Da un punto di vista pedagogico, il modello di riferimento è quello del learning by doing (imparare facendo): giocando scopro, penso, rifletto, formulo congetture, verifico, tento, ritento, argomento. I giochi promuovono comportamenti creativi e pensiero divergente; spesso agiscono come stimoli per l'apprendimento, inducendo una vivace discussione sui concetti di apprendimento tra gli studenti dopo il gioco (Boyle, 2011). Inoltre, I'elemento ludico permette allo studente di agire più spontaneamente e di muoversi in un ambiente a lui più confortevole e senza insidie.

Si ha così l'opportunità di proporre una didattica coinvolgente e di favorire un atteggiamento positivo verso la disciplina (Di Martino, 2007).

Nello specifico del percorso presentato, accanto al momento ludico, si propongono momenti di riflessione che stimolano l'argomentazione, la difesa della propria tesi, l'evoluzione della propria identità, in un terreno su cui lo studente si sposta con disinvoltura. L'evoluzione della competenza argomentativa degli studenti dalla Fase 1 alla Fase 3 si evince dalle loro produzioni. Basti confrontare le argomentazioni dei Gruppi 1, 2, 3 (par. 3) con quella di Gia. (par. 4), che descrive con precisione e buona capacità di sintesi le situazioni che si possono presentare, oppure con quella di Leonardo e Matteo:

«Per poter vincere bisogna puntare sulle seguenti caselle: 6-11-16, caselle che chiameremo

"Caselle Vincenti" queste ti porteranno alla vittoria!

Le caselle che bisogna evitare sono: 3-4-8-9-13-14-18-19, caselle che chiameremo "Caselle Perdenti" queste caselle sono assolutamente da evitare perché il tuo avversario potrebbe posizionarsi sulle caselle vincenti!

Un consiglio per vincere è quello di cominciare dalla casella numero 2 onde evitare che l'avversario possa raggiungere la casella numero 6 .

Se non è più possibile raggiungere le "Caselle Vincenti" una buona strategia è quella di puntare sulle caselle: 7-12-17, caselle che chiameremo "Caselle Semi vincenti"; quest'ultime potranno portarti alla vittoria, ma in alcuni casi porteranno ad un numero maggiore di 21 , il che corrisponderebbe a un pareggio, per questo abbiamo deciso di chiamarle "Semi vincenti"».

Le dimensioni del Quadro TRU trovano applicazione in questo percorso, in cui contenuti, coinvolgimento, sfide, quadro in continua evoluzione concorrono a creare quella identità matematica propria di ciascuno studente e di ciascun cittadino.

Con attività di questo tipo si ha l'occasione di presentare una matematica ancora in evoluzione, in cui ogni giorno si scoprono nuovi teoremi e in cui tutti gli studenti possono essere creativi e piccoli matematici: si presenta una matematica

«non ridotta a un insieme di regole da memorizzare e applicare, ma riconosciuta e apprezzata come contesto per affrontare e porsi problemi significativi e per esplorare e percepire relazioni e strutture che si ritrovano e ricorrono in natura e nelle creazioni dell'uomo».

(MIUR, 2012, p. 50)

\section{Ringraziamenti}

Ringraziamo la Professoressa Gabriella Chisari, dirigente del Liceo Scientifico Galileo Galilei di Cata- 
nia, e la Professoressa Agnese Zuccarello, docente di Matematica nella sezione di Liceo Matematico, che hanno consentito la sperimentazione dell'attività alla presenza di uno degli autori. Un ringraziamento speciale ai meravigliosi ragazzi della $1^{\text {a }}$ O, anno scolastico 2017/2018.

La ricerca è stata supportata dal progetto di ricerca "Programma Ricerca di Ateneo UNICT 2020-22 linea 2, EEEP\&DLaD".

\section{Bibliografia}

Anichini, G., Arzarello, F., Ciarrapico, L., \& Robutti, O. (2004). Matematica 2003. Matteoni Stampatore.

Bartolini Bussi, M. G., Boni, M., \& Ferri, F. (1995). Interazione sociale e conoscenza a scuola: la discussione matematica. Rapporto tecnico n.21 NDR di Modena. Centro Documentazione Educativa.

Boyle, S. (2011). Teaching toolkit: An introduction to game based learning. UCD Teaching and Learning Resources. https://www.ucd.ie/t4cms/UCDTLT0044.pdf.pdf

Bramanti, M., \& Travaglini, G. (2009). Matematica. Questione di Metodo. Zanichelli.

Brousseau, G. (1997). Theory of Didactical Situations in Mathematics. Kluwer Academic.

Capone, R., Rogora, E., \& Tortoriello F. S. (2017). La matematica come collante culturale nell'insegnamento. Matematica, Cultura e Società. Rivista dell'Unione Matematica Italiana, 2(3), 293-304.

Chiappini, G. (2007), Il laboratorio didattico di matematica: riferimenti teorici per la sua costruzione. In R. Garuti, A. Orlandoni \& R. Ricci (A cura di), Il laboratorio matematico scientifico: suggerimenti ed esperienze, allegato al n.8 di Innovazione educativa (pp. 9-12). IRRE Emilia Romagna.

Di Martino, P. (2007), L'atteggiamento verso la matematica: alcune riflessioni sul tema. L'insegnamento della matematica e delle scienze integrate, 30A-B(6), 651-666.

Ministero dell'istruzione, dell'università e della ricerca. (2012). Indicazioni nazionali per il curricolo della scuola dell'infanzia e del primo ciclo di istruzione. http://www.indicazioninazionali.it/2018/08/26/indicazioni-2012/

Ponte, J. P. (2001). Professional narratives in mathematics teacher education. Proceedings of the 2001 Annual Meeting of the Canadian Mathematics Education Study Group (pp. 61-65). University of Alberta. https:// www.researchgate.net/publication/277239646 Professional narratives in mathematics teacher education

Sawyer, B. (2007). The "Serious Games" Landscape. Proceedings of Instructional \& Research Technology Symposium for Arts, Humanities and Social Sciences. Camden.

Schoenfeld, A. H. (2013). Classroom observations in theory and practice. ZDM-The International Journal Of Mathematics Education, 45, 607-621.

Schoenfeld, A. H. (2014). What makes for powerful classrooms, and how can we support teachers in creating them? Educational Researcher, 43(8), 404-412.

Schoenfeld, A. H., \& the Teaching for Robust Understanding Project. (2016). An Introduction to the Teaching for Robust Understanding (TRU) Framework. Graduate School of Education. http://map.mathshell.org/trumath.php

Zirawaga, V. S., Olusanya, A. I., \& Maduku, T. (2017). Gaming in Education: Using Games as a Support Tool to Teach History. Journal of Education and Practice, 8(15), 55-64. 